\title{
Status of Light as Solution to Dark Energy
}

\section{Louise Riofrio}

Researcher, Insight Optics, Waimea, USA

Email: Louise.Riofrio@gmail.com

How to cite this paper: Riofrio, L. (2021) Status of Light as Solution to Dark Energy. Journal of Applied Mathematics and Physics, 9, 2579-2591.

https://doi.org/10.4236/jamp.2021.911166

Received: July 16, 2021

Accepted: October 31, 2021

Published: November 3, 2021

Copyright (c) 2021 by author(s) and Scientific Research Publishing Inc. This work is licensed under the Creative Commons Attribution International License (CC BY 4.0).

http://creativecommons.org/licenses/by/4.0/

\begin{abstract}
The original purpose of this research is to understand the origin and evolution of the Universe. I describe a simple cosmology that sheds light on the appearance of "dark" energy. Results explain the uniformity of the cosmic microwave background, critical density, and other phenomena. Light continues to expand our understanding of the "dark" Universe, with applications to the microscopic quantum world.
\end{abstract}

\section{Keywords}

Cosmology, Speed of Light, Quantum Gravity, Relativity

\section{Introduction}

Today's cosmology faces problems both early and late, models of the early Universe that cannot be tested, and data indicating that the late Universe accelerates. These problems have been previously been explained by a repulsive "inflation" and another repulsive "dark" energy. Recent studies cast doubt upon these speculations. A cosmology described by simple equations may bring light to this darkness.

This research began with an attempt to combine the local conditions of Special Relativity, which does not include gravity, with the curved Universe of General Relativity. A principle behind Special Relativity is that Space and Time are one phenomenon related by $c$. The factor $c$, experienced as the speed of light, can be considered a conversion factor between Space/Time [1]. From this principle, known as causality, the equations of Special Relativity can be derived [2].

Over the last century, many measurements show that the Universe has a finite age $t$, currently estimated at $13.8 \mathrm{Gyr}$. The Universe appears to have no centre in Space, but there is a centre in Time, an origin called the "Big Bang". Our separation from the Big Bang is simply age $t$.

This suggests that the expanding Universe is curved with radius given by: 


$$
R=c t
$$

where $R$ is the radius of curvature, $c$ is the speed of light.

\section{A Simple Equation and the Cosmic Horizon}

Many experiments today indicate that the Universe expands, but not why. Equation (1) explains the cosmological arrow of time, why scale $R$ expands as time $t$ increases. The Universe can't expand at the same rate continually, for gravity slows expansion. Factor $c$ must then be further related to $t$.

We have several ways of reaching Equation (5). One involves combining the rest and potential energy of a particle so that total energy is 0 .

$$
E+U=0
$$

where $E$ is rest (Einstein) energy, $U$ is potential (Newton) energy.

We then have:

$$
m c^{2}-G M m / R=0
$$

Here $m$ is mass of a test particle, though this would apply also to massless photons.

Inserting $R=c t$, we can remove $m$ :

$$
\begin{gathered}
G M m / c t=m c^{2} \\
G M=t c^{3}
\end{gathered}
$$

where $G$ is the gravitational constant, $M$ is mass of the Universe.

By this equation, when $t$ was tiny $c$ was enormous and $R$ expanded in a "Bang".

As time $t$ increases, $c$ slows and continues to decrease at a small rate today. This immediately sheds light on cosmology's "horizon problem". The microwave background temperature appears uniform to one part in $10^{4}$, indicating that distant parts of the early universe were within light's reach. The CMB surrounds us with evidence that the early speed of light was much faster.

An oft-repeated explanation has been that when the Universe was just $10^{-33}$ sec old it "inflated" many times faster than light. More than 40 years after it was first suggested, this old idea has proven cumbersome and unworkable. "Inflation" would violate both the First Law of Thermodynamics (Conservation of Energy) and Relativity's stipulation that nothing expands faster than $c$. To support this speculation, and a fixed $c$, theorists must invoke imaginary "inflatons" or "scalar fields" which cannot be found in nature.

The popular views of researchers such as Steinhardt [3], Ijjas [4], and Loeb [5] call into doubt whether "inflation" was ever a viable theory [6]. These authors question whether it is testable of falsifiable. Counter-arguments rely on an appeal to authority, claiming that if a group of theorists believe "inflation" then it must be right. A larger number have called for a better model of the early Universe.

The inflated idea was thought to be untestable, but is today unsupported by 
data. Observers cannot time-travel to the moments after the Big Bang, and no conceivable experiment can approach its titanic energies. However, most inflated ideas predict that B-mode polarization should appear in the CMB. The complete lack of B-modes after decades of search eliminates many inflated models [7].

\section{Curvature of Space/Time}

Every inflated model insists that the Universe is geometrically flat. CMB data has been called consistent with a flat geometry, but Sarkar et al. show that the data is more consistent with curvature [8]. Inflationary models uniformly insist that the scale of temperature fluctuations should be the same at all angles. Repeated analysis by Starkmann and colleagues [9] shows that these fluctuations are near zero for angles greater than 60 degrees, indicating curvature and disfavouring inflated ideas.

Recent analysis of Planck spacecraft CMB data by Di Valentino et al. shows an enhanced gravitational lensing amplitude, which is explained by positive curvature. This interpretation of Planck data favours curvature at a 3.4 $\sigma$ level [10]. A curved Universe, also was found by Refs. [8] and [9], would eliminate any inflated model.

We can calculate $c$ and radius of curvature $R$ as functions of time.

$$
\begin{gathered}
c(t)=(G M)^{1 / 3} t^{-1 / 3} \\
R(t)=c t=(G M)^{1 / 3} t^{2 / 3}
\end{gathered}
$$

From Equation (6) we integrate how far light from time $t=0$ has travelled.

$$
\begin{aligned}
& \int_{0}^{t_{0}} c(t) \mathrm{d} t=\int_{0}^{t_{0}}(G M)^{1 / 3} t^{-1 / 3} \\
& \int_{0}^{t_{0}} c(t) \mathrm{d} t=\frac{3}{2}(G M)^{1 / 3}\left(t_{0}\right)^{2 / 3}
\end{aligned}
$$

From $G M=t c^{3},(G M)^{1 / 3}=c_{0}\left(t_{0}\right)^{1 / 3}$

$$
\int_{0}^{t_{0}} c(t) \mathrm{d} t=\frac{3}{2} c_{0} t_{0}
$$

where $t_{0}$ is present time, $c_{0}$ is present value of $c$.

When the Universe is pictured as a globe of radius $R=c_{0} t_{0}$, we are on one pole and light from the Big Bang originates near the equator, traveling a distance $(3 / 2) c_{0} t_{0}$ to reach our telescopes. From our vantage point at the pole, two acoustic peaks predicted to be one degree apart will appear one degree apart on the Equator. The CMB shows that the Universe is curved with radius $R=c t$, as predicted.

Three entirely independent calculations-the scale of temperature fluctuations, enhanced lensing amplitude, and the distance light from the CMB has traveled-all show curvature of Space/Time. The last calculation shows that it is curved with the predicted radius $R=c t$. These independent pieces of evidence all argue against inflated models of a flat Universe. 


\section{A Stable Density}

Cosmology has another "flatness" problem, why overall density appears close to the critical value known as $\Omega$. Inflated models must begin at a density extremely close to $\Omega$, for the smallest deviation would be multiplied by inflated expansion. No inflated model explains this critical density. Rather than explain the "flatness" problem, "inflation" exponentially magnifies it.

We define $e$ as the deviation from critical density [11].

$$
\begin{gathered}
e=\Omega-1 \\
\dot{e}=(1+e) e\left(\frac{\dot{R}}{R}\right)(1+3 w)+2\left(\frac{\dot{C}}{c}\right) e
\end{gathered}
$$

where $\dot{e}$ is the time-derivative of $e$, the hypothetical $w=0$.

From Equation (6) and Equation (7) we have:

$$
\begin{gathered}
\frac{\dot{R}}{R}=\frac{2}{3 t} \\
\frac{\dot{c}}{c}=-\frac{1}{3 t} \\
\dot{e}=(1+e) e\left(\frac{2}{3 t}\right)-2\left(\frac{1}{3 t}\right) e \\
\dot{e}=e^{2}\left(\frac{2}{3 t}\right)
\end{gathered}
$$

As $t$ increases $\dot{e}$ and $e$ are both driven to 0 . The critical density is in fact the stable density, uniquely solving the "flatness" problem.

In this cosmology total energy $E$ of the Universe is 0 , but it can't be 0 everywhere. Quantum fluctuations cause energy levels to vary in Space/Time. In the early Universe overdense regions would collapse into singularities and disappear from our view, appearing voids between galaxies. As was predicted in 2004, normal Gaussian distribution tells how much that proportion is:

$$
\Lambda=68.3 \%
$$

This unique prediction has now been verified by both WMAP and Planck spacecraft.

\section{Exploding Supernova Data}

This finally leads to the problem of "dark" energy, why the Universe has appeared to accelerate. Low redshifts of objects increase linearly with distance, indicating expansion. High redshifts of Type Ia supernovae have appeared to increase non-linearly, leading to speculation about acceleration due to a repulsive "dark" energy [12]. Supernovae are the only evidence of acceleration [13]. After over 20 years of speculation nothing like "dark" energy can be found in nature.

Guffanti and colleagues, after surveying 740 supernovae, conclude that the evidence for acceleration is marginal [14]. Tutusaus et al. write that time-evolution in the luminosity of supernovae is a more likely explanation, fitting data better 
than "dark" energy [15]. The most recent analysis by Sarkar and colleagues at Institut d'Astrophysique shows convincingly that cosmic "acceleration" is premature [16]. Yet another analysis by Lee et al. shows that "dark" energy is not necessary [17]. To save the credibility of supernova data, another explanation is needed.

Redshift $Z$ is defined as:

$$
\frac{R_{0}}{R_{i}}=1+Z=\sqrt{\frac{1+\frac{v}{c}}{1-\frac{v}{c}}}
$$

where $v$ is an object's radial velocity, $Z$ is redshift.

From Equations (6) and (7) we have:

$$
\begin{aligned}
& \frac{R_{0}}{R_{i}}=\left(\frac{t_{0}}{t_{i}}\right)^{2 / 3}=1+Z \\
& \frac{c_{i}}{c_{0}}=\left(\frac{t_{0}}{t_{i}}\right)^{1 / 3}=\sqrt{1+Z}
\end{aligned}
$$

where $R_{0}$ and $c_{0}$ are their present values, $R_{i}$ and $c_{i}$ are their values at time $t_{i}$ when light was emitted.

The factor $\sqrt{1+Z}$ is negligible for low values of $Z$, but becomes noticeable for high-redshift supernovae. An object of redshift $Z=1$ recedes at $60 \%$ of today's speed of light $c_{0}$. By Equation (20), that is $42 \%$ of $c_{i}$ at time light was emitted. The redshift we observe is only 0.57 .

Supernova energy $E=m c^{2}$ would then be doubled for magnitude change of -0.75 . This precisely predicts the "accelerating" curve of Type Ia supernova redshifts (Figure 1) without any repulsive "dark" energies. Sanejouand [18] also writes that a varying speed of light explains the "accelerating" data.

\section{Galaxies, Sun and Moon}

A faster speed of light in the early Universe solves many problems. It would explain how supermassive Black Holes formed within galaxies, including our Milky Way. Old models of cosmology cannot explain how our galaxy and its central Black Hole formed. If $c$ were faster, supermassive Black Holes could have formed primordially from quantum density fluctuations.

The speed of light also explains the "Faint Young Sun" paradox. According to astrophysics models of the Sun, life cannot have evolved on Earth because $4 \mathrm{Gyr}$ ago the Sun shone with only $75 \%$ its present luminosity, and Earth's surface temperature would have been below the freezing point of water. Since the Sun turns its fuel to energy by $E=m c^{2}$, if $c$ has changed in exactly the amount predicted by $G M=t c^{3}$, solar luminosity would be almost exactly today's value. If the speed of light had not changed by this amount, primitive life forms could not insist that $c$ is fixed.

The most striking evidence of $c$ change today comes from our Lunar Laser 


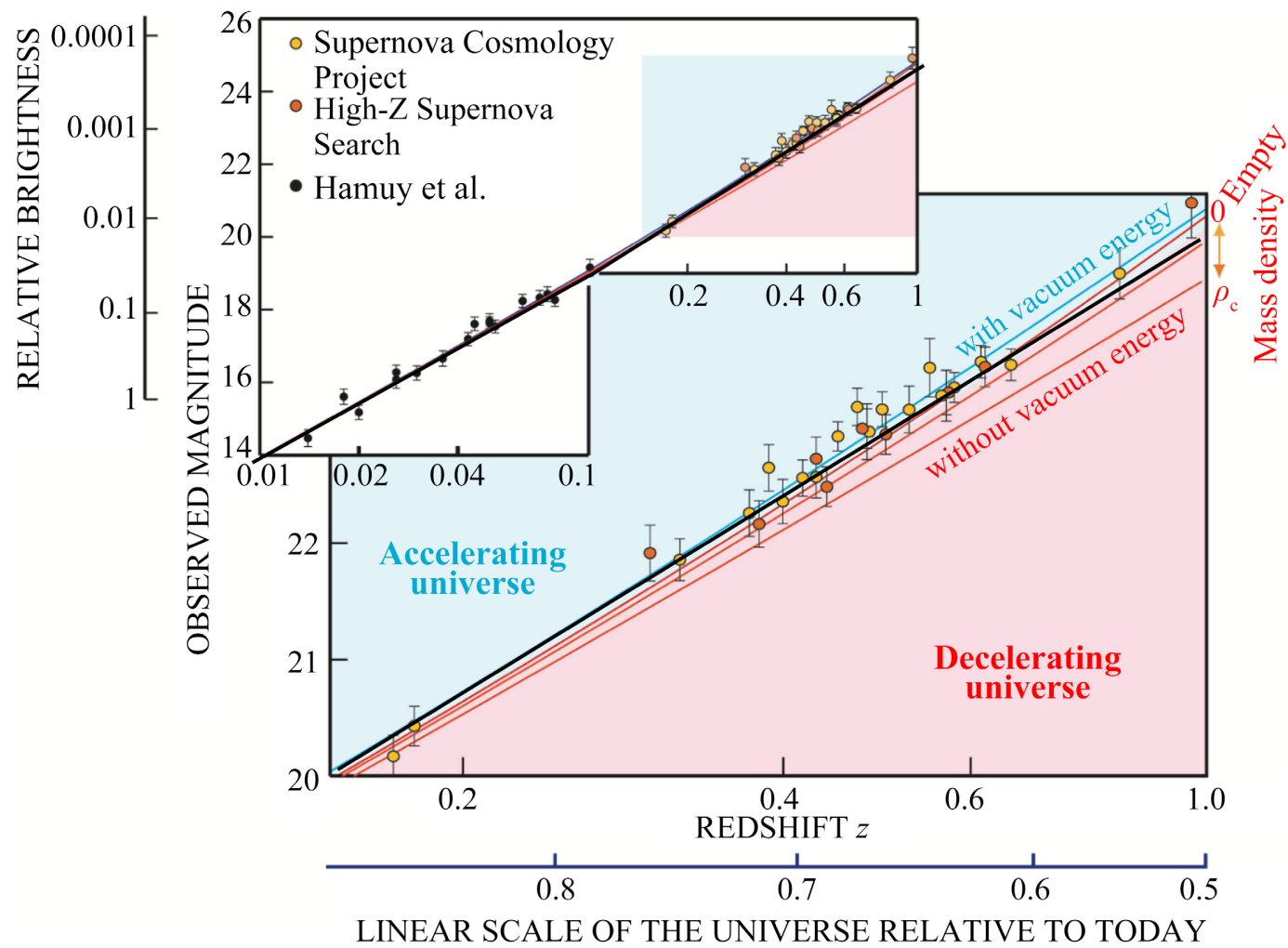

Figure 1. Prediction (thick black line) superimposed on data, courtesy supernova cosmology project.

Ranging Experiment. Since being placed on the Moon by Apollo astronauts, LLRE has allowed the lunar distance to be measured precisely. This $384,402 \mathrm{~km}$ semi-major axis has long been known to be increasing due to tidal interaction between Earth and Moon. LLRE has reported the semi-major axis increasing by $3.82 \pm 0.07 \mathrm{~cm} / \mathrm{yr}$, anomalously high. If the Moon were receding at this rate today, it would have been in the same orbital location as Earth just $1.5 \mathrm{Gyr}$ ago [19].

Independent experiments agree on a much lower recession rate. One dataset comes from tidal rhythmites, fossilized sediments left by ancient tides. The Mansfield Sediment of Indiana, the most extensively studied, reports a lunar distance of $375,300 \pm 1900 \mathrm{~km}$ at a time $310 \mathrm{Myr}$ ago. This translates to a recession rate of $2.9 \pm 0.6 \mathrm{~cm} / \mathrm{yr}$.

A second dataset comes courtesy of historical astronomers, the solar eclipse record. When the narrow track of an eclipse is recorded over an observatory, it provides a precise measure of Earth-Moon interaction. This method can determine lunar recession rate with an accuracy rivaling LLRE.

A recession rate of $3.82 \pm 0.07 \mathrm{~cm} / \mathrm{yr}$ corresponds to a change in Earth length-of-day $2.30 \pm 0.04 \mathrm{msec} / \mathrm{cyr}$. Eclipse observations spanning $2700 \mathrm{yrs}$, compiled by Stephenson and colleagues [20] show change in LOD of only $1.78 \pm$ $0.03 \mathrm{msec} / \mathrm{cyr}$. This corresponds to a recession rate of $2.96 \pm 0.05 \mathrm{~cm} / \mathrm{yr}$. LLRE differs by over 12 standard deviations, an extremely significant anomaly.

A third dataset comes from computer simulation. Poliakow [21] produced a 
detailed numerical model of Earth-Moon tidal interaction accounting for ocean depth, location of ocean basins, and movement of continents over time. This computer model predicts today's recession rate as $2.91 \mathrm{~cm} / \mathrm{yr}$. Three independent datasets agree, yet LLRE's laser light differs.

If the speed of light were slowing today, time for laser light to return from the Moon would be affected, causing LLRE's recession rate to appear larger. The discrepancy is proportional to $\dot{c} / c$.

From Equation (6) we have:

$$
\begin{gathered}
\frac{\dot{c}}{c}=-\frac{1}{3 t}=-\frac{\dot{a}}{a} \\
\dot{a}=\frac{a}{3 t}=\frac{384402 \mathrm{~km}}{3 \times 13.8 \mathrm{Gyr}} \\
\dot{a}=0.926 \mathrm{~cm} / \mathrm{yr}
\end{gathered}
$$

where $a$ is orbital semi-major axis, $\dot{a}$ is the predicted anomaly [22].

This predicts the 12-sigma anomaly within less than a standard deviation, over $99 \%$ certainty, indicating that speed of light $c$ is slowing at the predicted rate. Drifts in values such as $c$ may soon be tested by our Atomic Clock Ensemble in Space aboard the International Space Station. ACES is designed to detect variation of less than $10^{-10}$, so verifying $c$ change may be only a matter of time.

At one time it was possible to profess that the Universe was accelerating due to a repulsive "dark" energy. Measurements of the Hubble value from the CMB and the "accelerating" data from supernovae appeared to agree. Today they differ; the Hubble value from supernovae differs from the CMB result by $9 \%$. This has led to a crisis in cosmology when old models no longer agree with data.

Dr. Hebden writes that the crisis is explained if the early speed of light were greater [23]. This would cause high-redshift supernova data, which shows "acceleration," to show a higher expansion rate. If the speed of light slows, the industry of "dark" energy would no longer be necessary. Data should be examined in the context of a changing speed of light.

\section{Summary}

Darkness cannot drive out darkness, only light can do that.-Martin Luther King.

We have agreement from many researchers that old ideas of "inflation" and "dark" energy are unworkable. A cosmology accounting for the speed of light may be the only solution to the appearance of cosmic "acceleration". It also corrects deficiencies in inflated models of the early Universe. With new data, we may finally bring light to the great darkness of cosmology.

\section{Acknowledgements}

Great thanks are due to work done at NASA and Johnson Space Center, Houston. 


\section{Conflicts of Interest}

The author declares no conflicts of interest regarding the publication of this paper.

\section{References}

[1] Riofrio, L. (2004) GM $=\mathrm{tc}^{3}$ Space/Time Explanation of Supernova Data. Proceedings of the Beyond Einstein Conference, Palo Alto, 12-14 May 2004, 1-3. https://www-conf.slac.stanford.edu/einstein/talks/aspauthor2004_3.pdf

[2] Zeeman, E.C. (1964) Causality Implies the Lorentz Group. Journal of Mathematical Physics, 5, 490. https://doi.org/10.1063/1.1704140

[3] Steinhardt, P. (2011) The Inflation Debate: Is the Theory at the Heart of Modern Cosmology Deeply Flawed? Scientific American, 304, 36-43. https://doi.org/10.1038/scientificamerican0411-36

[4] Ijjas, A., Steinhardt, P. and Loeb, A. (2013) Inflationary Paradigm in Trouble after Planck 2013. Physics Letters B, 723, 261-266. https://doi.org/10.1016/j.physletb.2013.05.023

[5] Ijjas, A., Steinhardt, P. and Loeb, A. (2014) Inflationary Schism. Physics Letters B, 736, 142-146. https://doi.org/10.1016/j.physletb.2014.07.012

[6] Ijjas, A., Loeb, A. and Steinhardt, P. (2017) Pop Goes the Universe. Scientific American, 316, 32-39. https://doi.org/10.1038/scientificamerican0217-32

[7] Steinhardt, P. (2014) Big Bang Blunder Busts the Multiverse Bubble. Nature, 510, 9. https://doi.org/10.1038/510009a

[8] Sarkar, D., Huterer, D., Copi, C., Starkmann, G. and Schwarz, D. (2011) Missing Power vs. Low-L Alignments in the Cosmic Microwave Background: No Correlation in the Standard Cosmological Model. Astroparticle Physics, 34, 591-594. https://doi.org/10.1016/j.astropartphys.2010.12.009

[9] Copi, C., Huterer, D., Schwarz, D. and Starkmann, G. (2011) Large-Angle Anomalies in the CMB. Advances in Astronomy, 2010, Article ID: 847541. https://doi.org/10.1155/2010/847541

[10] Di Valentino, E., Melchiorri, A. and Silk, J. (2020) Planck Evidence for a Closed Universe and a Possible Crisis for Cosmology. Nature Astronomy, 4, 196-203. https://doi.org/10.1038/s41550-019-0906-9

[11] Albrecht, A. and Maguiejo, J. (1999) A Time-Varying Speed of Light as a Solution to Cosmological Puzzles. Physical Review D, 59, Article ID: 043516. https://doi.org/10.1103/PhysRevD.59.043516

[12] Perlmutter, S. (2003) Supernovae, Dark Energy and the Accelerating Universe. Physics Today, 56, 53. https://doi.org/10.1063/1.1580050

[13] Blanchard, A., Douspis, M., Rowan-Robinson, M. and Sarkar, S. (2003) An Alternative to the Cosmological "Concordance" Model. Astronomy \& Astrophysics, 412, 35. https://doi.org/10.1051/0004-6361:20031425

[14] Nielsen, J.T., Guffanti, A. and Sarkar, S. (2016) Marginal Evidence for Cosmic Acceleration from Type Ia Supernovae. Scientific Reports, 6, Article No. 35596. https://doi.org/10.1038/srep35596

[15] Tutusaus, I., Lamine, B., Dupays, A. and Blanchard, A. (2017) Is Cosmic Acceleration Proven by Local Cosmological Probes? Astronomy \& Astrophysics, 602, A73. https://doi.org/10.1051/0004-6361/201630289

[16] Colin, J., Mohayaee, R., Rameez, M. and Sarkar, S. (2019) Evidence for Anisotropy 
of Cosmic Acceleration. Astronomy \& Astrophysics, 631, L13. https://doi.org/10.1051/0004-6361/201936373

[17] Kang, Y.J., Lee, Y.-W., Kim, Y.-L., Chung, C. and Ree, C.H. (2020) Early-Type Host Galaxies of Type Ia Supernovae II: Evidence for Luminosity Evolution in Supernova Cosmology. Astrophysical Journal, 889, 8. https://doi.org/10.3847/1538-4357/ab5afc

[18] Sanejouand, Y. (2005) A Simple Varying Speed-of-Light Hypothesis Is Enough for Explaining High-Redshift Supernova Data. https://arxiv.org/abs/astro-ph/0509582

[19] Bills, B.G. and Ray, R.D. (1999) Lunar Orbital Evolution: A Synthesis of Recent Results. Geophysical Research Letters, 26, 3045-3048.

https://doi.org/10.1029/1999GL008348

[20] Stephenson, F.R., Morrison, L.V. and Hohenkerk, C.Y. (2016) Measurement of the Earth's Rotation: 720 BC to AD 2015. Proceedings of the Royal Society A, 472, 2196. https://doi.org/10.1098/rspa.2016.0404

[21] Poliakow, E. (2004) Numerical Modelling of the Paleotidal Evolution of the Earth-Moon System. Proceedings of the International Astronomical Union, Belgrade, Serbia and Montenegro, August 31-September 4, 2004, IAUC197, 445-452. https://doi.org/10.1017/S174392130400897X

[22] Riofrio, L. (2012) Calculation of Lunar Orbit Anomaly. Planetary Science, 1, 23 p. https://planetary-science.springeropen.com/track/pdf/10.1186/2191-2521-1-1.pdf https://doi.org/10.1186/2191-2521-1-1

[23] Hebden, K. (2019) As Mystery of the Universe's Expansion Rate Widens, a Simple Solution Is Offered. Room, 20, 88-91.

[24] Marshall, W.F., et al. (2012) What Determines Cell Size? BMC Biology, 10, 101. https://doi.org/10.1186/1741-7007-10-101

[25] Riofrio, L. (2019) An Exceptionally Simple Experiment Testing Quantum Theory and Gravity. Journal of Cosmology, 27.

[26] Consultative Committee for Units (2003) Report to the General Conference on Weights and Measures. International Bureau of Weights and Measures, Sevres 17-18 April 2003.

[27] Westphal, T., Hepach, H., Pfaff, J. and Aspelmeyer, M. (2021) Measurement of Gravitational Coupling between Millimeter-Sized Masses. Nature, 591, 225-228. https://doi.org/10.1038/s41586-021-03250-7

[28] Prescott, D.M. (1955) Relations between Cell Growth and Cell Division. Experimental Cell Research, 9, 2. https://doi.org/10.1016/0014-4827(55)90106-3

[29] Feric, M. and Brangwynne, C.P. (2013) A Nuclear F-Actin Scaffold Stabilizes Ribonucleoprotein Droplets against Gravity in Large Cells. Nature Cell Biology, 15, 1253-1259. https://doi.org/10.1038/ncb2830 


\section{Addendum: Applications to the Microscopic World}

As this article is being written, there is space to describe recent work about the microscopic quantum world. Though cosmology and origins of the Universe seem removed from everyday life, this recent work may apply to one of life's basic units, the cell. What limits cell size is a longstanding question of biology [24].

\section{A.1. Planck Values}

A changing $c$ suggests that the Planck value $h$ is linked. Speed of light $c$ and $h$ appear together in the fine-structure value, the photon energy and Chandrasekhar mass. While some data from quasars suggest that fine-structure value has changed, the value of $h c$ appears to be constant over time. If $c$ decreases then $h$ would decrease.

The increase proportional to $h \sim t^{2 / 3}$ is similar to the solution of a 3-dimensional random walk problem. This first link between Relativity and Quantum Mechanics may explain the thermodynamic "arrow of time" or why entropy increases. The uncertainties are given by $h$ increase over time, causing universal entropy to also increase.

Equations (1) and (5) may be further simplified using the units introduced by Max Planck, made of combinations of $h, c$, and $G$ :

$$
\begin{aligned}
& t_{P L}=\sqrt{\frac{\bar{h} G}{c^{5}}} \\
& l_{P L}=\sqrt{\frac{\bar{h} G}{c^{3}}} \\
& m_{P L}=\sqrt{\frac{\bar{h} c}{G}}
\end{aligned}
$$

where $t_{P L}$ is Planck time, $I_{P L}$ is length and $m_{P L}$ is mass.

Using Planck units, Equations (1) and (5) are combined:

$$
\begin{gathered}
M / m_{P L}=R / l_{P L}=t / t_{P L}=0.79 \times 10^{61} \\
M=R=t
\end{gathered}
$$

This extremely simple equation describes the large Universe in tiny Planck units.

The large number $0.79 \times 10^{61}$ is beyond the scope of a short paper to derive. When $h c$ is considered constant, Planck length increases as $I_{P L} \sim t^{2 / 3}$ in proportion to scale $R$ of the Universe, Planck time $t_{P L}$ would increase proportionally with age $t$. The Planck mass, $2.2 \times 10^{-8} \mathrm{~kg}$, appears fixed over time.

Equation (28) does tell us that the largest possible measures, size and age of the Universe, are multiples of the Planck units. It suggests that these units are fundamental, that Space/Time is discontinuous at the Planck scale. While the Planck time and length are too small to be measured, the Planck mass is a macroscopic quantity similar to a flea's egg or a human eyelash hair. Small masses are normally measured with mass spectrometers, but these devices measure inertial, not gravitational mass. 
Inertial mass is experienced as an object reacts to an accelerating force. Gravitational mass, according to General Relativity, causes Space/Time to curve affecting both other objects and the propagation of light. As shown by Galileo, Earth's gravity causes objects to fall toward Earth at the same rate. Two small particles dropped from Pisa's Leaning Tower fall toward the Piazza de Miracoli because of Earth's large mass.

Many people have seen dust fall toward Earth, and dust particles tend to settle uniformly on a flat surface without attracting one another. (The "dust bunnies" that gather in corners are a result of electrostatic and aerodynamic action). I have observed the fall of lunar dust particles, and their behaviour in a vacuum is similar. The observation that small particles do not attract each other is a clue to a quantized gravitational mass.

Equation (28) suggests that the Planck gravitational mass is fundamental, and masses below $m_{P L}$ do not cause curvature of Space/Time. Two tiny particles fall from the Leaning Tower because of Earth's large mass, but they do not attract each other gravitationally. The Equivalence Principle assumes that inertial and gravitational mass are always equal, but this assumption has not been tested for sub-Planck masses. The following experiment tests for quantized mass.

\section{A.2. Prediction}

If gravitational mass is quantized at the Planck scale, two sub-Planck masses will not attract each other.

\section{A.3. Procedure}

An exceptionally simple experiment, within the capability of any laboratory or classroom, can test for quantized gravitational mass. The nucleus of the experiment is two spherical sub-Planck masses. We have used two limestone spheres of diameter $0.2 \mathrm{~mm}$. With a limestone density of $2500 \mathrm{~kg} / \mathrm{m}^{3}$ each sphere has a mass of $1.0 \times 10^{-8} \mathrm{~kg}$, slightly less than Planck mass.

The two masses are placed $1 \mathrm{~mm}$ apart on a low-friction surface such as Teflon. The surface is kept precisely level to reduce the influence of Earth's downward gravitational pull. Grounding the masses via the surface prevents them from developing an opposing electric charge. The experiment is placed in a vacuum chamber to reduce the effects of atmospheric pressure.

\section{A.4. Preliminary Results}

Acceleration between two Planck masses would be, at a distance of $1.0 \mathrm{~mm}$ :

$$
a=G m_{P L} / r^{2}=\left(6.67 \times 10^{-11}\right)\left(2.2 \times 10^{-8}\right) /\left(10^{-3}\right)^{2}=1.4 \times 10^{-12} \mathrm{~m} / \mathrm{sec}^{2}
$$

where $r$ is distance, $a$ is gravitational acceleration between two masses.

Time to travel an observable distance of 100 microns may be approximated:

$$
t=(2 d / a)^{1 / 2}=1.2 \times 10^{4} \mathrm{sec}
$$

where $t$ is time to travel 100 microns, here $12,000 \mathrm{sec}$ or $200 \mathrm{~min}$. 
According to universal theories of gravity, the two spherical masses should roll toward each other. After long observation no motion can be detected. This hints that the sub-Planck masses do not produce curvature of Space/Time. Reproduction of the experiment by independent laboratories is most welcome [25].

\section{A.5. An Experiment in Space}

Our International Space Station makes many kinds of science possible. The orbital environment removes most effects of external gravity. Clocks in Space can be more accurate for measuring changes in quantities like $c$. A further refinement of the experiment could take place in orbit.

Here the two masses are enclosed in an evacuated nucleus $2 \mathrm{~mm}$ in diameter within a $16 \mathrm{~mm}$ polycarbonate sphere. When the two masses are within a spherical shell, gravity from the shell does not affect their motion. This version of the experiment, no larger than a marble, can be carried into orbit in an astronaut's personal effects. Aboard a space station, the experiment can be left in a corner and observed for signs of motion.

\section{A.6. Observations of Nature}

A quantized gravitational mass may be observed in nature, where the fall of small particles toward Earth and not toward one another is but one example. Gravity does not appear in the interactions of small masses, specifically atoms and subatomic particles. Measurements of the International Prototype Kilogram have been found inaccurate by one Planck mass or about $2 \times 10^{-8} \mathrm{~kg}$ [26]. This IPK anomaly is explained when gravitational mass is quantized at the Planck scale. Experiments of Westphal and colleagues [27] find gravitational attraction between masses down to the $10^{-5} \mathrm{~kg}$ level, and may someday be able to test Planck masses.

Tiny creatures are seen in a microscope tumble about and behave as if in microgravity. Without gravity's aid, they must extend pseudopods or simple tentacles to draw food toward them. One-celled life forms appear to inhabit a world free of self-gravity. The experiments of Brangwynne and colleagues find that gravity is the determining factor in cell size [28].

Larger eukaryotic life forms are composed of cells whose size is largely limited by the Planck mass [29]. The living cell, a basic unit of life, processes nutrients and stores genetic materiel to create new cells. The cell's activities appear independent of gravity. The size of cells is another hint that gravity is quantized at the Planck scale.

The largest cell of the human body is the female ovum, with mass slightly less than the Planck mass. Upon fertilization by the male sperm cell, the ovum must reproduce to create new cells and develop into an embryo. The chromatids, containing DNA, must migrate to opposite ends of the cell to begin the process of mitosis. If a cell were more massive than the Planck mass, self-gravity would affect its internal processes. 


\section{A.7. Conclusions}

A simple cosmology explains puzzles of the early and late Universe, and also may connect the large and the small. The simplified equation $M=R=t$ hints that the Planck units are fundamental. Observation of small particles hints that gravitational mass is quantized. A simple experiment indicates that sub-Planck mass particles do not attract each other gravitationally. Future experiments may investigate whether Planck time and length are indivisible.

This cosmology began with an attempt to combine Special and General Relativity. We may further link the large Universe of Relativity with the microscopic quantum world. Applications extend to the basic units of life, the size of human cells. We can bring light to these questions of nature, a triumph for our understanding. 